\title{
METAHEURISTIC OPTIMIZATION \\ BASED MULTI-KEY ENABLED ENCRYPTION WITH IMAGE CLASSIFICATION FOR BIG DATA ENVIRONMENT IN HEALTHCARE SECTOR
}

\author{
Avula Satya Sai Kumar \\ Research Scholar, Department of Computer Science and Engineering, \\ Annamalai University, Tamilnadu, India. \\ satya.avula@gmail.com \\ Dr. S. Mohan \\ Assistant Professor, Department of Computer Science and Engineering, \\ Annamalai University, Tamilnadu, India. \\ mohancseau@gmail.com \\ Dr. A.Nagesh \\ Professor, Dept. of CSE, Mahatma Gandhi Institute of Technology, Hyderabad. \\ anagesh_cse@mgit.ac.in
}

\begin{abstract}
In recent times, medical imaging domain undergoes significant development with respect to innovations, market growth, and exploitation with the rise in the generation of massive data quantity poses diagnostic imaging in the context of Big data. At the same time, securing medical images is needed and it remains a crucial process on the shared communication model. Encryption is considered an effective way of securing the data transmission process in big data environment. Besides, the computer aided diagnosis also provides a second opinion to professionals to manage parallelism. So, it becomes essential to design digital environments and applications which offer effective handling of medical images such as Big data. This paper presents a new Metaheuristic Optimization based Multi-Key Enabled Encryption with Image Classification (MOMEE-IC) for Big Data Environment in Healthcare Sector. The proposed model involves two major operations as encryption and image classification. Firstly, the encryption process involves Multiple key based Homomorphic Encryption (MHE) with lion optimization algorithm (LOA) based optimal key generation process, called MHE-LOA. Besides, Stacked Denoise Autoencoder with Logistic Regression (SDAE-LR) based image classification process is employed for diagnosing the images in the cloud platform. To ensure the superior results of the presented model, a wide set of simulations were performed and the results are examined under distinct aspects.
\end{abstract}

Keywords: Big data, Cloud computing, Security, Encryption, Image classification.

\section{Introduction}

Medical imaging based diagnostic models have become popular in the healthcare sector and the massive growth of medical images resulted in the generation of big data. The concept of big data defines a highly complicated dataset which is defined by the classical variables over time and is represented by V's. The initial V volume indicates the complete number of data components in the massive dataset describing the accumulation of data from distinct sources. Next, the second $\mathrm{V}$ velocity defines the high speed at which data is created and the next $\mathrm{V}$ veracity indicates the intrinsic data uncertainties, value denotes the worthiness of data being extracted is comprised [1]. Alternatively, less direct, descriptions define the unusual competence which is needed to manage and analyze massive data quantity. So, big data analytics techniques are particularly developed for resolving this issue. Instead of representing big data by various V's, [2] defined Big data effectually, which employs massive and heterogeneity data quantity comprising the independent sources with distributed and decentralized control and seeks to explore complicated and growing relationship between the data. It is noticed that reputed corporates like Facebook, have comprised the explosion of information by promising openly available projects and data sharing. 
In the medical sector, big data comprises objective and subjective discoveries, parametric and nonparametric metrics, generated from distinct sources by the use of incongruent sampling. These data are structured, concentrating on genotypes, proteomic data, or, unstructured, including memo, prescription, medicinal images, etc [3]. Radiologists and scientists attempt to develop models in the direction of connecting Big data in images, that have the ability to lead advanced medical provision, custom-made diagnosis procedures, and capability of optimizing patient output [4]. Big data analytics defines the way of examining massive data, identification of clusters and correlation among the dataset, designing predictive technique using the data mining approaches [5].

Security becomes a fundamental problem to handle the transmission of images and encryption is used to achieve secrecy in the communication process. Several data security solutions are available in the literature and encryption is found to be a commonly available technique. It converts the plain input image into a number-image using a technique so as to join the primary image with a minimum of single key. Encryption is the process of executing exceptional numerical techniques and keys for transforming digital data to cipher data prior to transmission. On the decryption side, the algorithm with identical keys is employed for recovering the images.

Recently, the research is moving towards the classification of medical images from the big data environment, which is not handled by classical models. The method of parallelizing the medical images through multiple nodes boosts the speed and competence of the system [6-8]. The parallel distributed processing of the massive quantity of data is handled by the use of Hadoop and MATLAB distributed computing server for raising the computational complexity of the nodes for data flow. In addition, some studies are trying to handle the real application concurrently in big data analytics [9, 10]. A brain tumor segmentation technique is validated by the use of a parallel genetic approach (PGA) in Hadoop and Spark environments under distinct sizes of data [11]. An effective model in the cloud platform called a proficient lossless compression technique is designed to transfer the brain imaging in the cloud setting is employed. A new 2D medical imaging examination particularly MR images takes place in the Hadoop MapReduce framework [12]. The goal of the study is to minimize the retrieval time of the MR images. The MapReduce has the ability of managing massive number of images compared to the classical content based medical image retrieval system (CBMRS) owing to the utilization of basic image processing techniques alone. But they are relevant to the evidence based Practice (EBP) that assist professional for handling persons with identical situations. A 2-stage model results to make decisions related to surgeries when the number of retrieved tumor infected images is high.

An automated 3D MR image diagnosis model is presented in [13] by the use of scale invariant feature transform (SIFT) as feature extractor in the image. In addition, the feature vectors undergo mapping with the individual input image by the use of MapReduce and afterward saved using Hadoop Distributed File System (HDFS). The application of MapReduce split the images that automated reduces the detection time, but, simplify the expert decision-making process. Few statistics based segmentation techniques are hidden Markov models (HMMs) used for training and segmenting brain tumors. The training of HMM model uses MapReduce for increasing the efficiency of the multimodal MR brain images.

This paper presents a new Metaheuristic Optimization based Multi-Key Enabled Encryption with Image Classification (MOMEE-IC) for Big Data Environment in Healthcare Sector. The presented model encompasses two functions namely encryption and image classification. Primarily, the MOMEE process uses Multiple keybased Homomorphic Encryption (MHE) with lion optimization algorithm (LOA) based optimal key generation process. Besides, Stacked Denoise Autoencoder with Logistic Regression (SDAE-LR) based image classification process is employed for diagnosing the images in the cloud platform. To ensure the efficiency of the presented technique, an extensive performance analysis takes place and the outcomes are examined under distinct aspects.

\section{The Presented MOMEE-IC Model}

The presented MOMEE-IC model involves two stages as encryption and image classification. Initially, the medical images are encrypted using the MHE-LOA algorithm where the optimal keys in the MHE method are generated using the LOA. Next, the encrypted images will be transmitted to the cloud using the big data environment. Upon reception, the decryption of medical images takes place, and then image classification process gets executed using SDAE-LR model. Fig. 1 shows the overall working process of proposed MOMEE-IC model. 


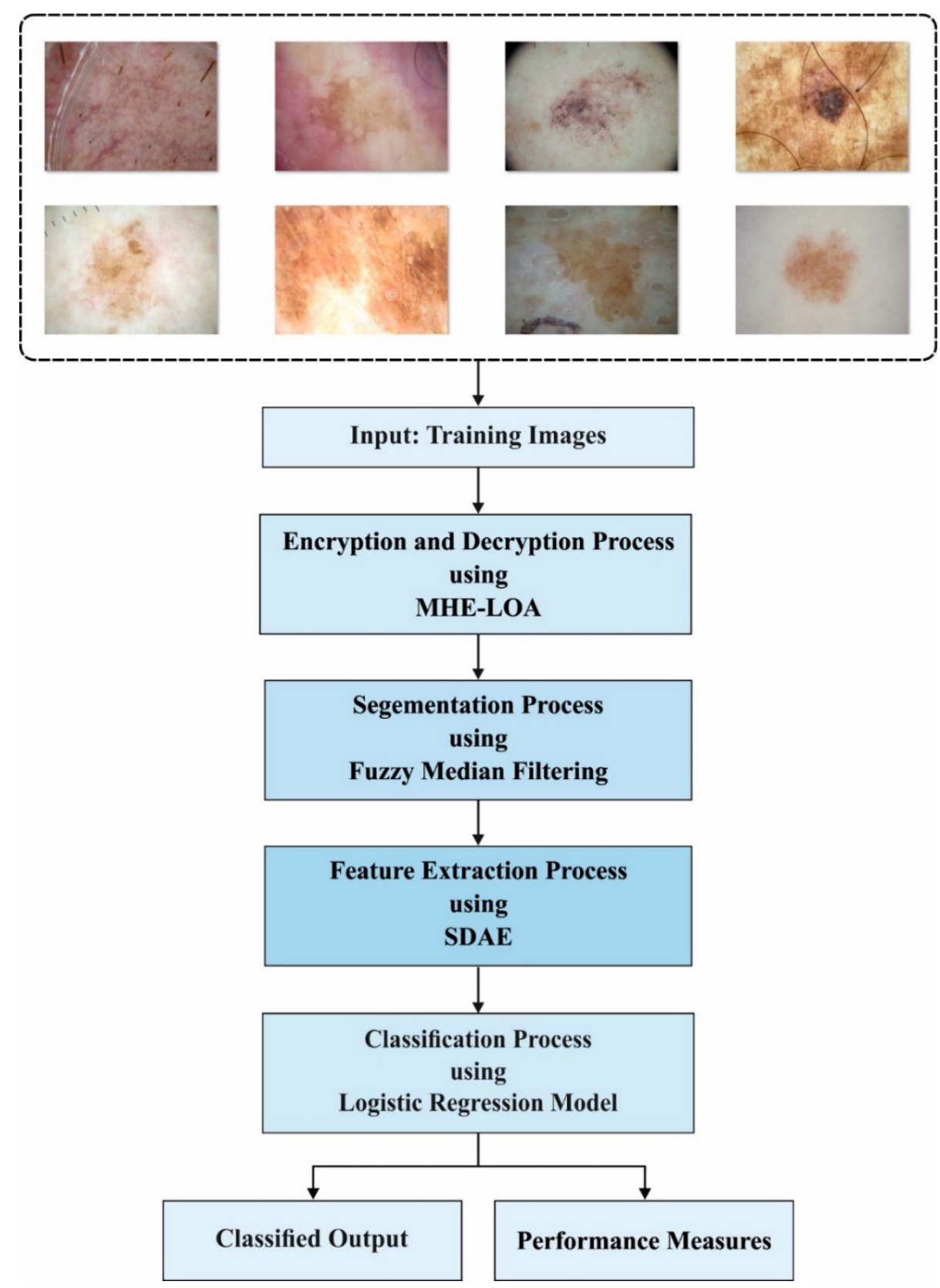

Fig. 1. Working process of MOMEE-IC model.

\subsection{MHE-LOA based Encryption Process}

The presented MHE-LOA model employs the Region of interest (ROI) of the input images to be split into an arbitrary number of blocks and are scuffled inside the image. The comprehensible data exist in the image owing to the correction that exists between the image components. To achieve secrecy, MHE process is applied and the encrypted data is generated. In addition, a set of public and private keys are utilized to perform the encryption and decryption process. Here, LOA is utilized for the generation of optimal keys to achieving improved security. Fig. 2 illustrates the process of MHE.

The ROI indicates the portion of an image where the filter performs certain operations on it. On the generated masked image, the pixel values which define the RoI are fixed to 1 and other pixel values are defined to 0 . Then, MHE technique is applied. A semantically secure homomorphic public key encryption method is utilized [14]. The feature of homomorphic is respected in the design of an encryption standard with highly secured data. They are used to carry out the tasks by the use of encoded data with no knowledge of the private key (without decryption). Homomorphic valuation technique considers the polynomial numerous cipher images, that undergo encryption using $\mathrm{N}$ keys and generates the cipher image. Multiple encryptions are an effective way of modifying input data to a scrambled form by executing encryption several times. It is defined by the processes of cascade encryption, cascade ciphering, and multiple encryptions. Here, the processes of encryption and decryption involve many generate and the optimal key generation process gets executed using LOA. 


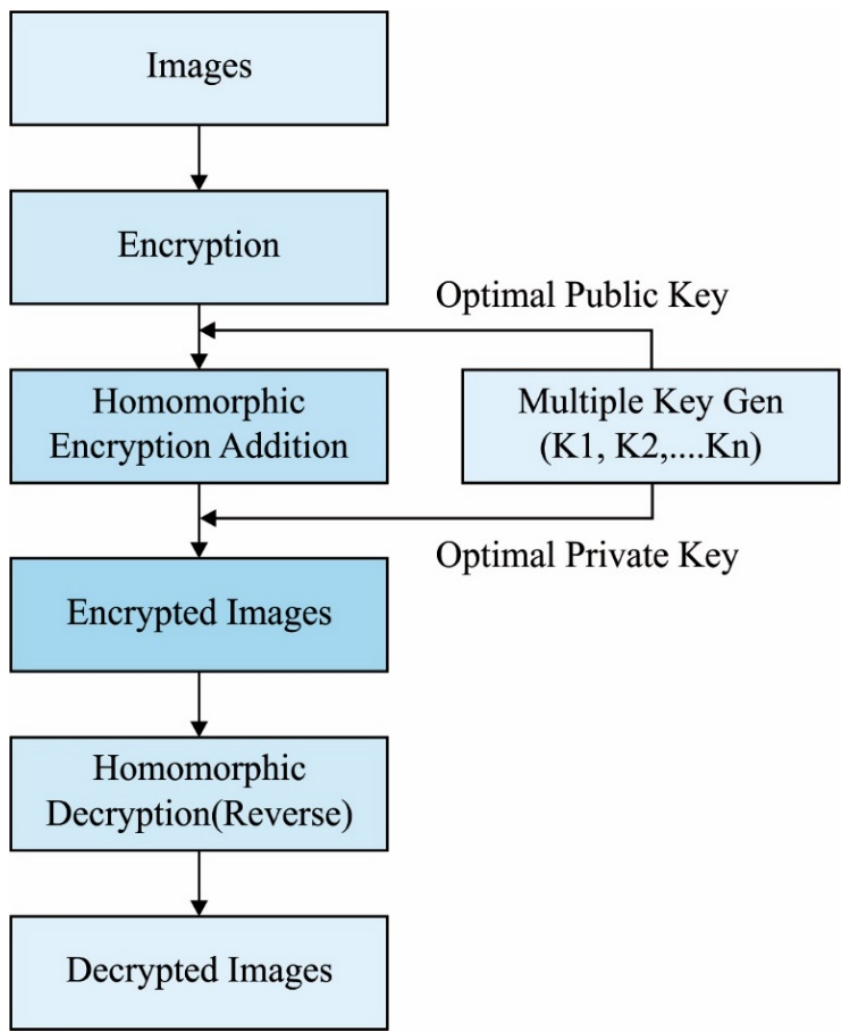

Fig. 2. Process flow of MHE.

Generally, keys are used for encrypting and decrypting the data. A key match is used with the asymmetric key technique where many keys $\mathrm{K}=\left\{\mathrm{K}_{1}, \mathrm{~K}_{2}, \ldots \ldots \mathrm{K}_{\mathrm{n}}\right\}$ are produced for MHE. In certain situations, the random generation of keys takes place by the use of a Random Number Generator. To select an optimum key from many keys, LOA is applied. LOA is simulated with the nature of lions [15]. A loin is generally depicted a superior level of collaboration as well as aggression. The lions are socially organized into 2 types such as resident lions (RL) and nomads (NL). In RL are generally live in families known as pride (P). The second type travels infrequently, either in sets or separately. Lions generally move to hunt in the procedure of groups. In several phases contained in the LOA are described under:

\section{Initialization}

In LOA is primarily creates the population arbitrarily in the solution space. An LOA regards all the individual solutions as a 'lion.' In $\mathrm{N}_{\mathrm{v}}$ dimensional optimization issue, the lion is determined as

$$
\text { Lion }=\left[\mathrm{u}_{1}, \mathrm{u}_{2}, \mathrm{u}_{3}, \ldots, \mathrm{u}_{\mathrm{N}_{\mathrm{v}}}\right]
$$

Then, the cost (fitness value (FV) of all the lions) is defined by estimation of cost function is provided under:

$$
\text { FV of lion }=f(\text { Lion })=f\left(u_{1}, u_{2}, u_{3}, \ldots, u_{N_{v}}\right)
$$

\section{Hunting}

At each $\mathrm{P}$, an assured amount of female appearances to the prey in the set to food the members in P. This hunter lion follows specific schemes to surround and catch the victim. In general, the lion follows nearly similar patterns during the hunting process. If the hunter improves the fitness, the prey gets escaped to a recent place as demonstrates in Eq. (3).

$$
P R Y^{\prime}=\mathrm{PRY}+\operatorname{random}(0,1) \times \mathrm{PRYI} \times(\mathrm{PRY}-\text { Hunter })
$$

where PRY is the current position of prey, Hunter is the new position of the hunter who attacks the prey and PRYI is the \% of improvement in the hunter's fitness. An updated place of the hunter which goes to left as well as right wings are produced as given below:

$$
\text { Huntr }^{\prime}=\left\{\begin{array}{cc}
\operatorname{random}((2 \times \mathrm{PRY}-\text { Huntr }), \text { PRY }), & (2 \times \mathrm{PRY}-\text { Huntr })<\mathrm{PRY} \\
\operatorname{random}(\mathrm{P},(2 \times \mathrm{PRY}-\text { Huntr })), & (2 \times \mathrm{PRY}-\text { Huntr })>\mathrm{PRY}
\end{array}\right.
$$

New positions of the Center hunters are produced as determined in Eq. (5):

$$
\text { Hunter }^{\prime}=\left\{\begin{array}{lc}
\text { random }(\text { Huntr, PRY }), & \text { Huntr }<\text { PRY } \\
\text { random(PRY, Huntr) }, & \text { Huntr }>\text { PRY }
\end{array}\right.
$$


Rand $(a, b)$ gives an arbitrary number among $a$ and $b$. The hunting performance gives few advantages to achieve optimal solutions. This method provides a circle shape neighborhood surround the prey, and creates the hunter to go nearer to prey in several ways.

\section{Move Towards Safer Place}

Thus the region of all the pride contains the personal best positions achieved by all the members in pride and benefits the LOA for storing the better results obtained before a duration that should be helpful to increases the solutions in LOA. Hence, a novel position for female lion (FL) is expressed as

$$
\begin{gathered}
\mathrm{FL}=\mathrm{FL}+2 \mathrm{D} \times \operatorname{random}(0,1)\{\mathrm{R} 1\}+\mathrm{U}(-1,1) \times \tan (\theta) \times \mathrm{D} \times \operatorname{random}(0,1)\{\mathrm{R} 2\} \\
\{\mathrm{R} 1\} .\{\mathrm{R} 2\}=0,|| \mathrm{R} 2||=1
\end{gathered}
$$

where FL is the current place of FL, D provides the distance among the FL's position as well as chosen point selected by the tournament chosen in the pride region. $\{\mathrm{R} 1\}$ is a vector that indicates the primary point is the earlier place of the FL, and it is focused on the elected position. $\{\mathrm{R} 2\}$ is perpendicular to $\{\mathrm{R} 1\}$.

\section{Roaming}

In all the male lions (ML) in $P$ roam surrounded the region of the pride. In order to mimic the behavior, the $\%$ $\mathrm{R}$ of pride region is randomly elected and is stayed by that lion. As roaming, if the resident male determines an updated place which is optimal to the current place, the best-visited solution obtains upgraded. The NL and the adjustable roaming behavior use LOA for exploring the solution space in an arbitrary method and evade get trapped into the local optima. In this procedure, new positions of the NL are determined as

$$
\text { Lion }_{i j}^{\prime}=\left\{\begin{array}{lc}
\text { Lion }_{i j} & \text { if }>p r_{i} \\
R A N D_{j} & \text { otherwise }
\end{array}\right.
$$

where Lion $_{i}$ denotes the current place of the ith NL lion, $j$ indicates a dimension, rand $_{j}$ refers a uniform arbitrary number in the interval of $[0,1], R A N D$ refers an arbitrarily generated vector in the search space, and $p r_{i}$ represented the probability which is independently calculated to all NL lions as expressed under:

$$
\operatorname{pr}_{\mathrm{i}}=0.1+\min \left(0.5, \frac{\left(\mathrm{NWL}_{\mathrm{i}}-\mathrm{Best}_{\mathrm{NWL}}\right)}{\text { Best }_{\mathrm{NWL}}}\right)
$$

Where $\mathrm{NWL}_{\mathrm{i}}$ and Best ${ }_{\mathrm{NWL}}$ are the cost of current positions of the ith lion in NL and the better cost of the NL lion, correspondingly.

\section{Mating}

It is an important process which provides assurance to the being of lions and providing an opportunity for communicating to members. At each P, \%Ma of FLs undergo mating with several resident males that are elected arbitrarily from the similar P to create offsprings.

\section{Defense}

In each $\mathrm{P}$, if the ML obtains mature, it is violent as well as fights through other MLs in P. The beaten lion leaves the P and develops NL. Simultaneously, if an NL male is bodily strong, it fights through RL male in P. The defense function uses LOA to retain influential MLs as solutions that show an important portion in LOA.

\section{Migration}

According to the switch and migrate operations, it performs lions in one $\mathrm{P}$ to another or modifies its routine life and the resident female become NL, and conversely, it enhances the variety of a target pride with its position in the previous pride. In each $\mathrm{P}$, the highest count of females is calculated by $\mathrm{S} \%$ of the population of $\mathrm{P}$. To migration function, and a few females are arbitrarily elected and develop NL. If the selection FLs migrated in P and developed NL, new as well as old NL females are organized depends on the FV. Fig. 3 demonstrates the flowchart of LOA technique.

The fitness function involved in the MHE-LOA technique is the PSNR with high reconstructed image quality. The fitness function is defined as follows.

$$
\mathrm{F}_{\mathrm{i}}=\operatorname{Max}(P S N R)
$$

Homomorphic encryption technique encrypts the plain image and the cipher image is handled with an identical algebraic function. It allows the server to perform some actions on the encrypted data with no knowledge about the primary plain image. By the use of the secret keys, the user can encrypt the input image and generate $p r_{k}$ and $\mathrm{pu}_{\mathrm{k} \text {-optimal }}$ and, alongside public key $\left(p u_{k}\right)$, the cipher image is transmitted to the cloud $\mathrm{pu}_{\mathrm{k}}=(\mathrm{k}, \mathrm{i})$ and $\mathrm{K}=$ $(\mathrm{p}, \mathrm{q}) \operatorname{Enc}\left(\mathrm{I}, \mathrm{pr}_{\mathrm{k}}\right)$ to select an arbitrary variable $\mathrm{r} \in \mathrm{Z}_{\mathrm{k}}^{*}$, then the cipher data can be computed as $c=I \cdot r^{k} \bmod k^{2}$.

At the decryption process, the cipher image with encrypted pixels is resolved by ( $p, q)$ and the Secret vector. The decryption process involves a pair of masks namely secret and even Masks in a stable development. For decrypting the image, the respective keys are used and effective reconstruction of images takes place. 


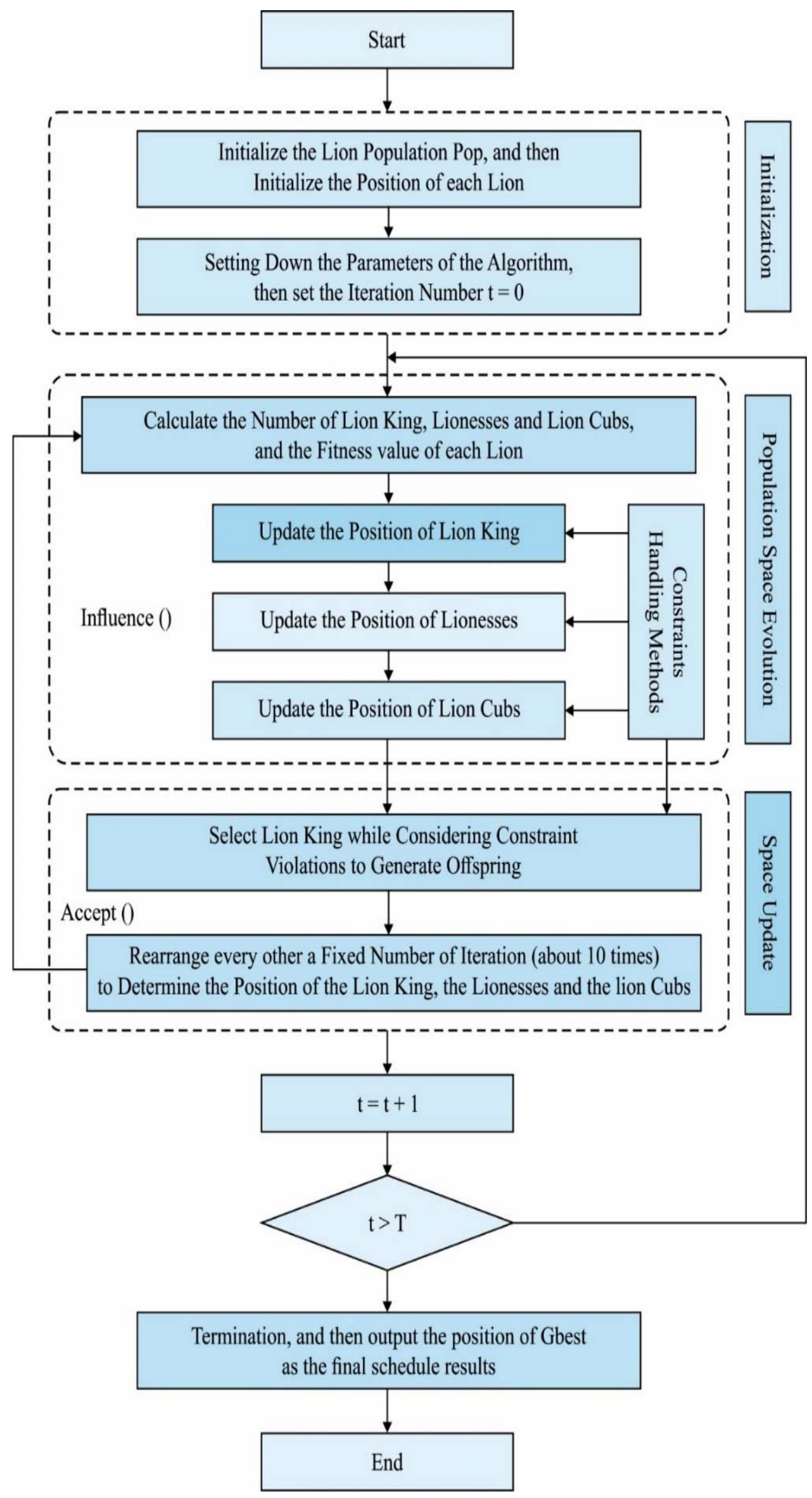

Fig. 3. Flowchart of LOA algorithm. 


\subsection{SDAE-LR based Image Classification Process}

At this stage, the decrypted images are classified using the SDAE-LR model. In addition, fuzzy mean filtering (FMF) technique is applied to segment the affected regions from the medical image and then features are extracted using SDAE model. At last, the LR model is applied as a classifier to allocate proper class labels to the applied input images.

\subsubsection{FMF based Segmentation}

The fuzzy set [16] is one of the unsupervised clustering techniques utilized to segment the medical image. It offers uniformly balanced data and rapid process of selecting the threshold value of the images at the time of training. To achieve precise clusters, it is employed on all slices of the medical images individually.

$$
\text { Fuzzy }_{\text {set }}=\sum_{x=1}^{N} \sum_{y=1}^{C} \operatorname{mean}_{i j}^{m}\left\|k_{x}-c_{y}\right\|^{2} 1 \leq \text { set }<\infty
$$

where set indicates $>1$ real numbers, $\left(\right.$ mean $\left._{i j}\right)$ denotes the degree of membership $k_{x}$ in y cluster. $k_{x}$ is determined using $d$-dimension $i_{\text {th }}$ data, $c_{y}$ is center of $d$-dimensional cluster, and $\|*\|$ denotes the resemblance among the center and measured data. The mean $_{i j}$ membership and cluster center $c_{y}$ are upgraded using the following equations.

$$
\begin{array}{r}
\operatorname{mean}_{i j}=\frac{1}{\sum_{k=1}^{y}\left(\frac{\left\|k_{x}-c_{y}\right\|}{\left\|k_{x}-c_{y}\right\|}\right)^{\frac{2}{\text { set }-1}}} \\
c_{y}=\frac{\sum_{y=1}^{N} \text { mean }_{x y}^{\text {set }} \cdot k_{x}}{\sum_{y=1}^{N} \text { mean }_{x y}^{\text {set }}}
\end{array}
$$

This procedure gets repeated until $\max _{x y}\left\{\left|\operatorname{mean}_{x y}^{(n+1)}-\operatorname{mean}_{x y}^{(n)}\right|\right\}<\delta$ where $\delta$ represents the stopping criteria among 0 to 1 , and $n$ indicates the iteration count. It gets converged into a saddle point or local minima of Fuzzy $_{\text {set }}$. Furthermore, morphological operation is carried out for refining the areas of tumor. They are utilized for removing additional pixel values, and therefore, the segmented image $g_{(x, y)} \mathrm{P}$ is tuned. The disk shape structuring component $g^{B(p, q)}$ with radius 4 is employed. Also, the dilation operation is employed to redefine the missing pixel values. The outcome of the segmentation process is determined as follows.

$$
\begin{aligned}
& \left(g_{(x, y)} \oplus g^{B}\right)(l, m)=\max _{p, q}\left(g_{(x, y)}(l-p, m-q)+g^{B}\right) \\
& \left(g_{(x, y)} \ominus g^{B}\right)(l, m)=\min _{p, q}\left(g_{(x, y)}(l-p, m-q)+g^{B}\right)
\end{aligned}
$$

Typeset sub-subheadings in medium face italic and capitalize the first letter of the first word only. Section numbers to be in roman.

\subsubsection{SDAE-LR based Classification}

DAEs are stacked for designing a deep network with multiple hidden layers. The SDAE model comprises 2 encoding as well as decoding layers. At the encoder side, the outcome of the preceding encoder acts as the input of the succeeding encoder. Assume that there exist $L$ hidden layers in the encoder, the activation function of the $k$ th encoding layer is defined here.

$$
\mathrm{y}^{(k+1)}=f_{e}\left(\mathrm{~W}^{(k+1)} \mathrm{y}^{(k)}+\mathrm{b}^{(k+1)}\right), k=0, \ldots, L-1,
$$

where the $\mathrm{y}^{(0)}$ indicates the actual data $\mathrm{x}$. The outcome $\mathrm{y}^{(L)}$ of the final encoding layer indicates the high level features filtered using SDAE model. At the decoder side, the outcome of the initial decoder layer is treated as the input of the subsequent decoding layer. The decoder function of the $k$ th decoder layer can be defined as follows. is

$$
\mathrm{z}^{(k+1)}=f_{d}\left(\mathrm{~W}^{(L-k) T} \mathrm{z}^{(k)}+\mathrm{b}^{\prime}(k+1)\right), k=0, \ldots, L-1,
$$

where the input $\mathrm{z}^{(0)}$ of the primary decoder layer is the outcome $\mathrm{y}^{(L)}$ of the previous encoding layer. The outcome $\mathrm{z}^{(L)}$ of the final decoder layer is the rebuilding of the actual data $\mathrm{x}$. In training procedure of the SDAE can be represented below.

1. Select the input data that are arbitrarily chosen from the medical images.

2. Perform training of the initial DAE that comprises the initial encoder layer and the final decoder layer. Attain the network weight and bias $\mathrm{W}^{(1)}$ and $\mathrm{b}^{(1)}$, and the features $\mathrm{y}^{(1)}$ are given as the outcome of the initial encoding layer. 
3. Utilize $\mathrm{y}^{(k)}$ as the input data of the $(k+1)$ th encoder layer. The $(k+1)$ th DAE can be trained and attain $\mathrm{W}^{(k+1)}$ and $\mathrm{b}^{(k+1)}$ and the features $\mathrm{y}^{(k+1)}$, where $k=1, \ldots, L-1$ and $L$ denotes the hidden layer count in the network.

It is found that the DAEs undergo training autonomously and then the training of SDAE is named layer-wise training. Furthermore, the trained network weights using SDAE act as the initialized weights in the subsequent LR fine-tuning stage [17]. So, the SDAE performs network pretraining.

\section{SDAE-LR}

Next, the SDAE-LR comprises SDAE pretraining and fine-tuning of the LR model. The SDAE performs training of the network weights and attains features using reconstruction-oriented learning, and the learned weights are replaced as the initial weights of the network. It can be notable that the SDAE is an unsupervised model and LR is a supervised model which involves only labeled data. The structure of SDAE-LR model is shown in Fig. 4. It includes a 2-class classification problem where the outcome will be two choices. The decoder of the SDAE can be eliminating and the encoder of the SDAE is kept for generating the first features. Besides, the final output layer of the entire network is known as the LR layer and the sigmoid activation function of the LR layer is defined below.

$$
h(\mathrm{x})=\frac{1}{1+\exp (-\mathrm{Wx}-\mathrm{b})},
$$

where $\mathrm{x}$ is the outcome $\mathrm{y}^{(L)}$ of the previous encoding layer. It includes the deeper features which are pretrained by the SDAE technique. The outcome of the sigmoid function ranges from 0 to 1 denoting the classifier outcome. The labels are linked to the training data points, and the error amongst the predicted and actual class labels are used to tune the network weight. Besides, the cross-entropy based cost function can be represented as

$$
\text { Cost }=-\frac{1}{m}\left[\sum_{i=1}^{m} l^{(i)} \log \left(h\left(\mathrm{x}^{(i)}\right)\right)+\left(1-l^{(i)}\right) \log \left(1-h\left(\mathrm{x}^{(i)}\right)\right)\right]
$$

where $l^{(i)}$ signifies the labels of the instance $\mathrm{x}^{(i)}$. The network weights are updated by the minimization of the cost function.

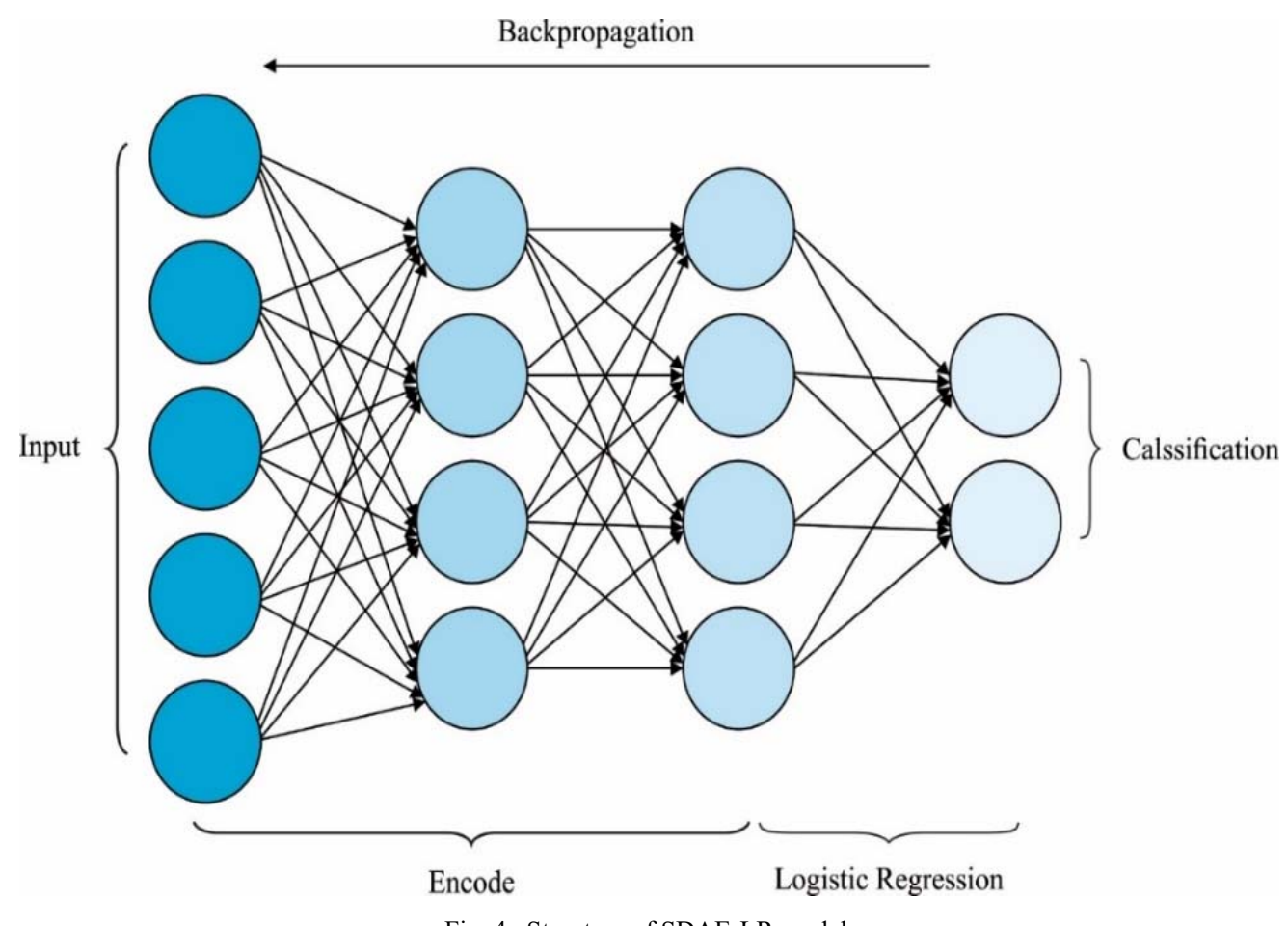

Fig. 4. Structure of SDAE-LR model.

The processes involved in the training of the SDAE-LR model are given below.

1. SDAE is used for training the primary network weights.

2. The weights of LR are arbitrarily initialized.

3. The training dataset is utilized as input and the predicted classifier outcome is generated using the initial weights of the entire network.

4. Network weight will be set using an iterated way by the minimization of the cost function in (9) by the use of MSGD technique. 
Next to the network training, the features of the input are computed and are considered as the outcome of the final encoding layer. The features are then fed to the LR model which provides output as the appropriate class label of the input. So, the processes of feature extraction and classification take place parallel.

\section{Performance Validation}

The performance validation of the MHE-LOA model takes place using ISIC skin lesion imaging dataset. Few sample images from the dataset are shown in Fig. 5.
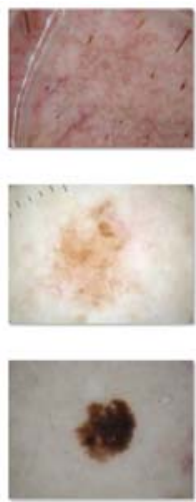
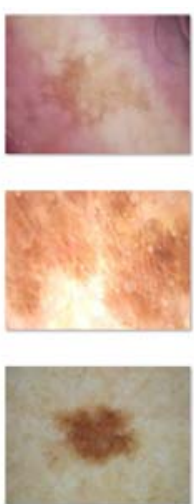
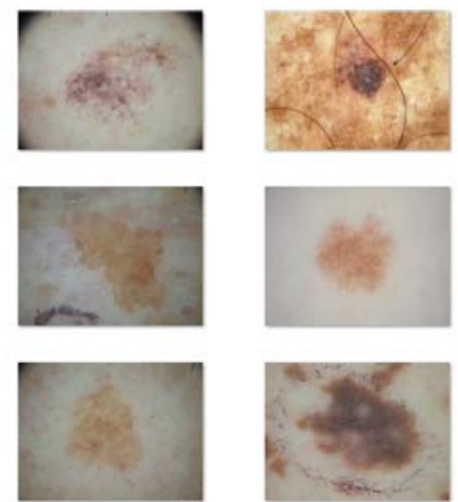

Fig. 5. Sample Images.

Table 1 visualizes the encryption results of the MHE-LOA technique on the applied different skin lesion images. The first column depicts the input image, second column shows the encrypted image and the last column represents the decrypted image. These images signified that the MHE-LOA model has effectively encrypted the input and decrypted it without any loss of information.

Table 1. Result Analysis of Proposed Encryption Method MHE-LOA.

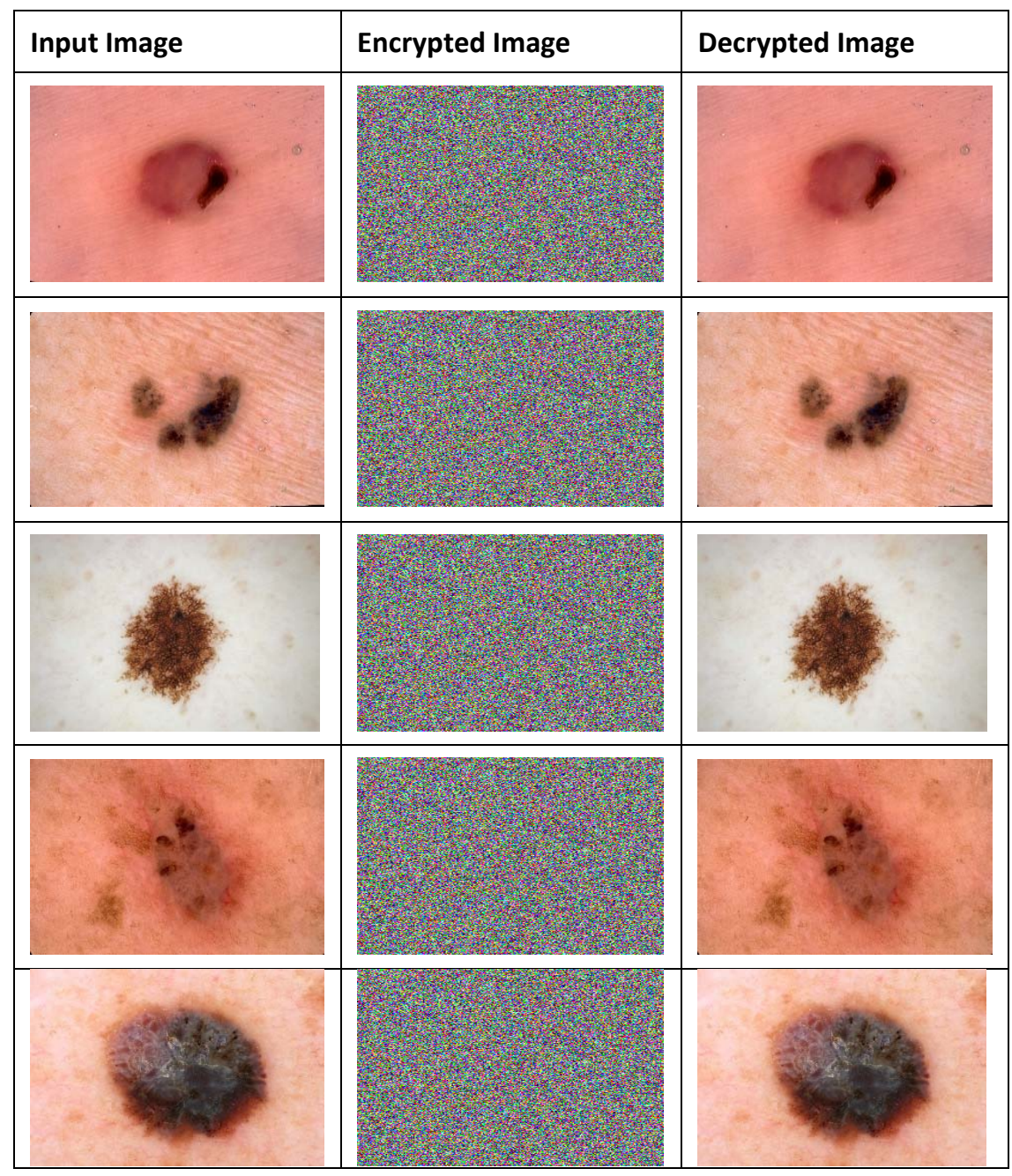


Table 2 gives a comprehensive encryption results analysis of the MHE-LOA model with existing methods [18]. Fig. 6 investigates the MSE and RMSE analysis of the MHE-LOA model. The figure referred that the HE model has shown inferior encryption outcome by obtaining highest MSE of 0.6903 and RMSE of 0.8308 . At the same time, the MHE model has shown slightly enhanced outcomes by offering an MSE of 0.3437 and RMSE of 0.5863. Similarly, the MHE-AWO model has showcased reasonable outcomes with the MSE of 0.0933 and RMSE of 0.3055 . But the presented MHE-LOA model has displayed effective outcomes by achieving the least MSE of 0.0494 and RMSE of 0.2223 .

Table 2. Result Analysis of Existing with Proposed MHE-LOA Method in terms of Different Measures.

\begin{tabular}{|l|l|l|l|l|}
\hline Methods & MSE & RMSE & PSNR & Corr.Coef \\
\hline MHE-LOA & 0.0494 & 0.2223 & 61.194 & 0.9963 \\
\hline MHE-AWO & 0.0933 & 0.3055 & 58.432 & 0.9532 \\
\hline MHE & 0.3437 & 0.5863 & 52.769 & 0.9365 \\
\hline HE & 0.6903 & 0.8308 & 49.740 & 0.9178 \\
\hline
\end{tabular}

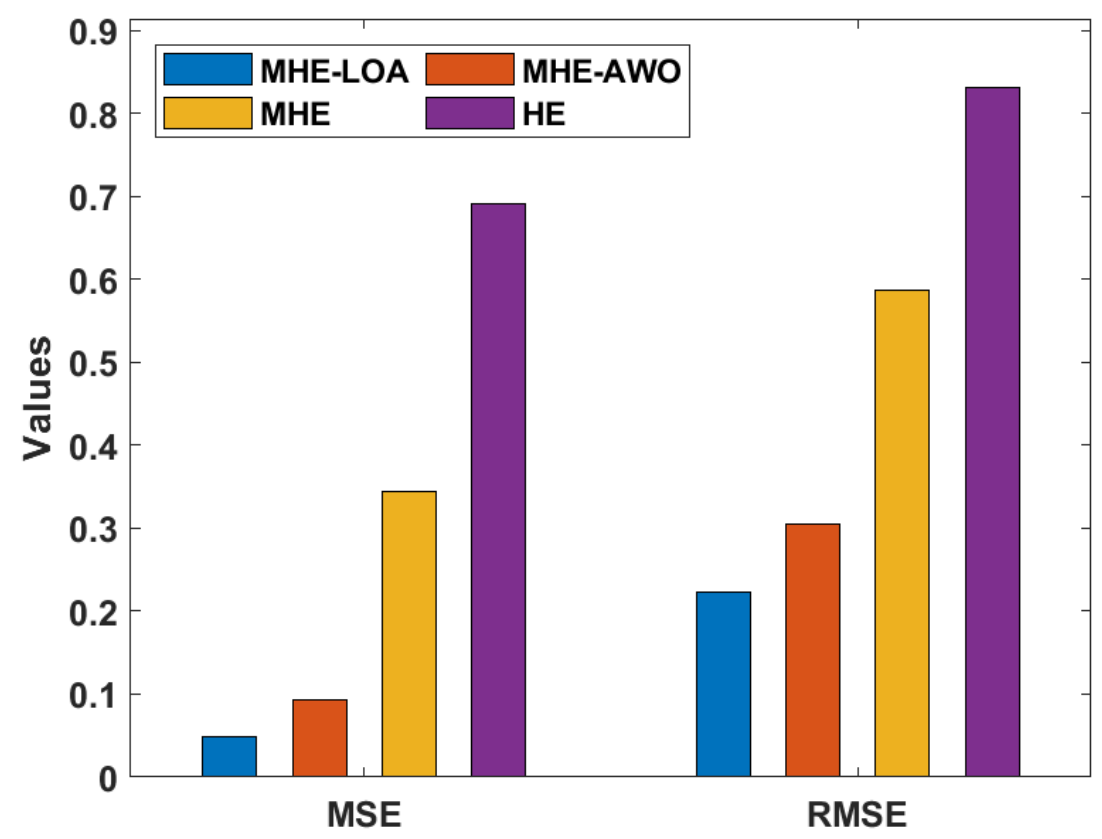

Fig. 6. MSE and RMSE analysis of MHE-LOA model.

Fig. 7 performs the PSNR analysis of the MHE-LOA with existing methods. The figure stated that the HE model has accomplished lower performance and achieved a lower PSNR of $49.740 \mathrm{~dB}$ whereas a slightly increased PSNR of $52.769 \mathrm{~dB}$ has been offered by the MHE model. Along with that, the MHE-AWO model has demonstrated a moderate PSNR of 58.432dB. At last, the MHE-LOA model has resulted in a higher PSNR of 61.194dB. 


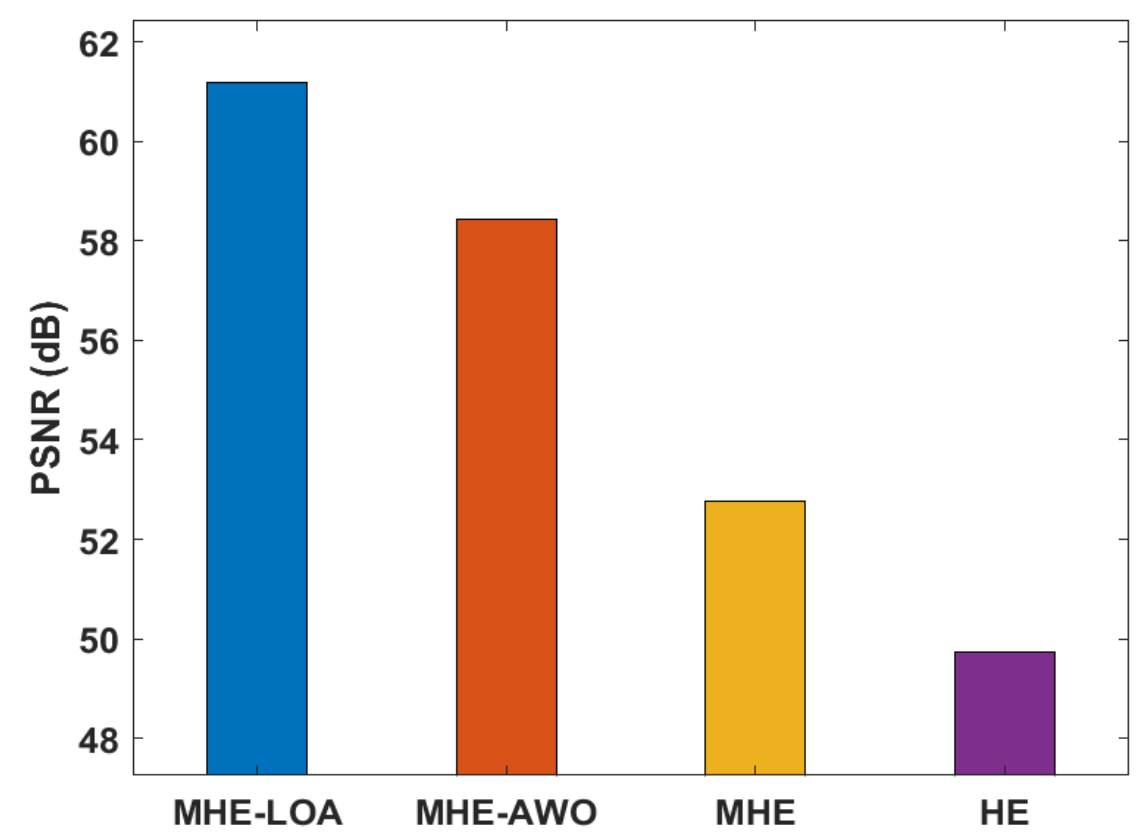

Fig. 7. Result analysis of MHE-LOA model interms of PSNR.

Fig. 8 examines the $\mathrm{CC}$ analysis of the MHE-LOA with existing techniques. The figure represented that the HE model has accomplished worst performance and achieved a minimum CC of 0.9178 whereas a slightly improved CC of 0.9365 has been offered by the MHE technique. Likewise, the MHE-AWO method has exhibited a moderate CC of 0.9532. Finally, the MHE-LOA methodology has resulted in a superior CC of 0.9963.

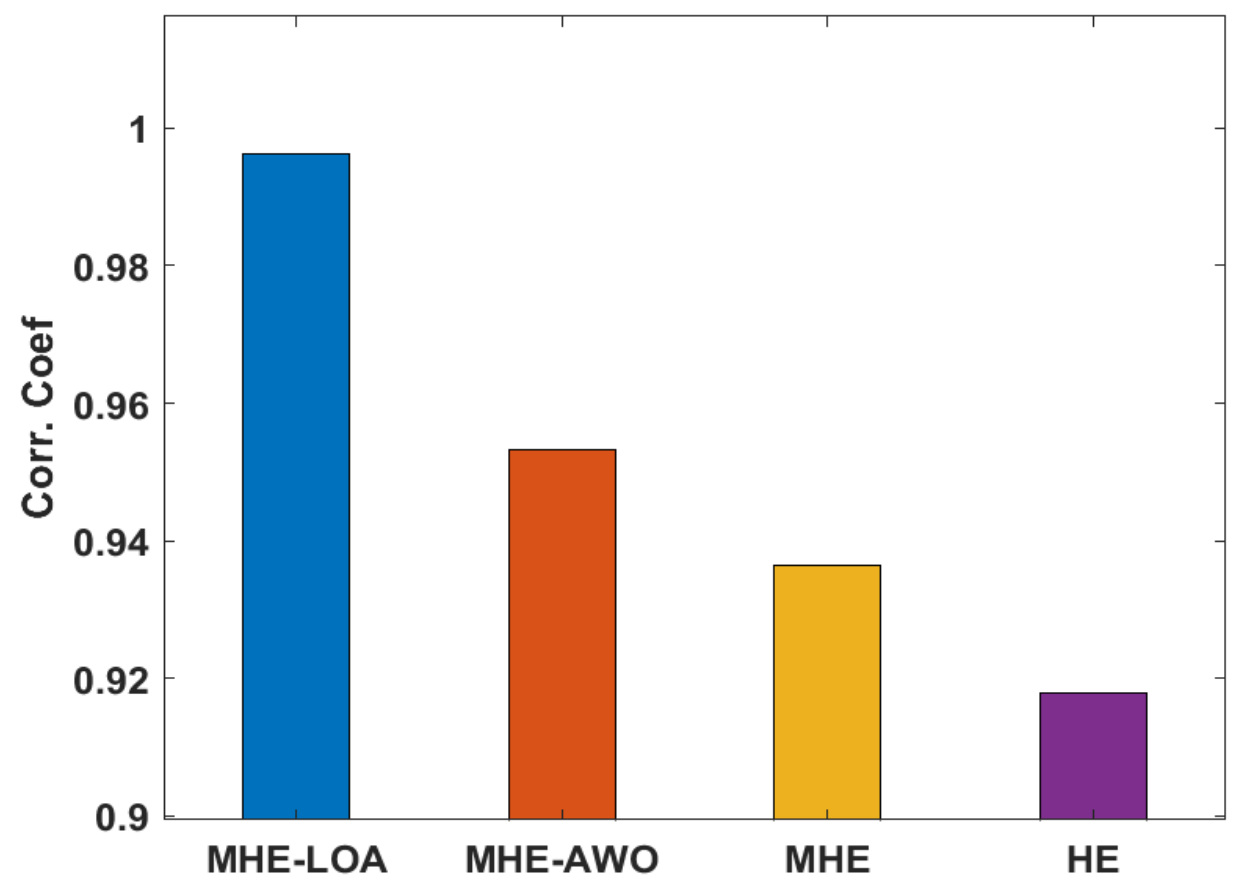

Fig. 8. Result analysis of MHE-LOA model interms of CC.

Table 3 depicts a complete comparative classification results analysis of the MOMEE-IC method interms of distinct measures [19]. Fig. 9 demonstrates the accuracy analysis of the MOMEE-IC method with the state of art techniques. From the figure, it can be apparent that the ResNet 50 model has resulted in lower accuracy of $75.5 \%$. Likewise, the VGG19-SVM approach has achieved a slightly increased accuracy of $80.7 \%$ whereas an even improved accuracy of $81.2 \%$ has been obtained by the VGG19 model. Besides, the Li et al. method has displayed moderate results with an accuracy of $93.2 \%$ whereas a closer accuracy of $93.39 \%$ has been offered by the Halil et al. method. Followed by, the Yuan et al. method has accomplished manageable accuracy of 93.4\% whereas a certainly enhanced accuracy of $94.03 \%$ is demonstrated by the AI-Magni et al. method. Though the DL-ANFC model has resulted in a near optimal accuracy of $97.91 \%$, the presented MOMEE-IC model has depicted better outcome with a maximum accuracy of $98.69 \%$. 
Table 3. Result Analysis of Existing with Proposed MOMEE-IC Method in terms of Different Measures.

\begin{tabular}{|l|l|l|l|}
\hline Methods & Sensitivity & Specificity & Accuracy \\
\hline MOMEE-IC & 94.82 & 98.95 & 98.69 \\
\hline DL-ANFC & 93.40 & 98.70 & 97.91 \\
\hline Halil et al., & 90.82 & 92.68 & 93.39 \\
\hline AI-Magni et al., & 85.40 & 96.69 & 94.03 \\
\hline Li et al., & 82.00 & 97.80 & 93.20 \\
\hline Yuan et al., & 82.50 & 97.50 & 93.40 \\
\hline ResNet50 & 90.00 & 61.00 & 75.50 \\
\hline VGG19 SVM & 93.00 & 69.00 & 80.70 \\
\hline VGG19 & 95.00 & 68.00 & 81.20 \\
\hline
\end{tabular}

Fig. 10 illustrates the sensitivity and specificity analysis of the MOMEE-IC method with existing models. From the figure, it can be apparent that the Li et al. model has resulted in a minimum sensitivity of $82 \%$. Similarly, the Yuan et al. approach has obtained a somewhat improved sensitivity of $82.5 \%$ whereas an even higher sensitivity of $85.4 \%$ has been attained by the AI-Magni et al. method. Also, the ResNet-50 method has showcased moderate results with a sensitivity of $90 \%$ whereas a closer sensitivity of $90.82 \%$ has been offered by the Halil et al. technique. At the same time, the VGG19-SVM model has accomplished manageable sensitivity of $93 \%$ whereas a certainly improved sensitivity of $93.4 \%$ is exhibited by the DL-ANFC method. However, the proposed MOMEE-IC methodology has resulted in a near optimal sensitivity of $94.82 \%$, the VGG19 algorithm has outperformed better results with the higher sensitivity of $95 \%$.

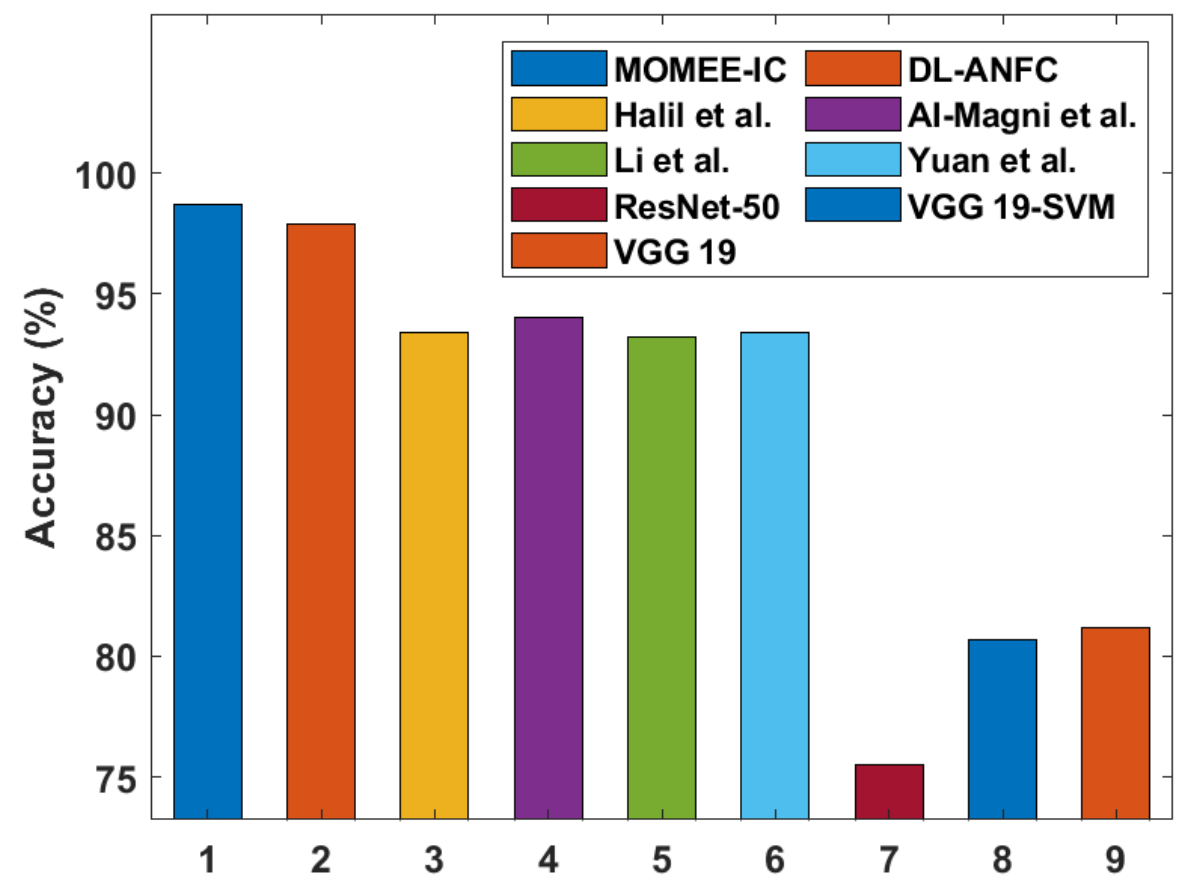

Fig. 9. Comparative analysis of MOMEE-IC Method interms of Accuracy.

From the figure, it is apparent that the ResNet 50 approach has resulted in a lesser specificity of $61 \%$. In line with this, the VGG19 technique has reached a somewhat higher specificity of $68 \%$ whereas an even enhanced specificity of $69 \%$ has been achieved by the VGG19-SVM model. Along with that, the Halil et al. algorithm has portrayed moderate outcomes with the specificity of $92.68 \%$ whereas a closer specificity of $96.69 \%$ has been offered by the AI-Magni et al. technique. Additionally, the Yuan et al. approach has accomplished manageable specificity of $97.5 \%$ whereas a certainly higher specificity of $97.8 \%$ is depicted by the Li et al. method. But, the DL-ANFC technique has resulted in a near better specificity of $98.7 \%$, the projected MOMEE-IC technique has demonstrated optimal results with the superior specificity of $98.95 \%$. 


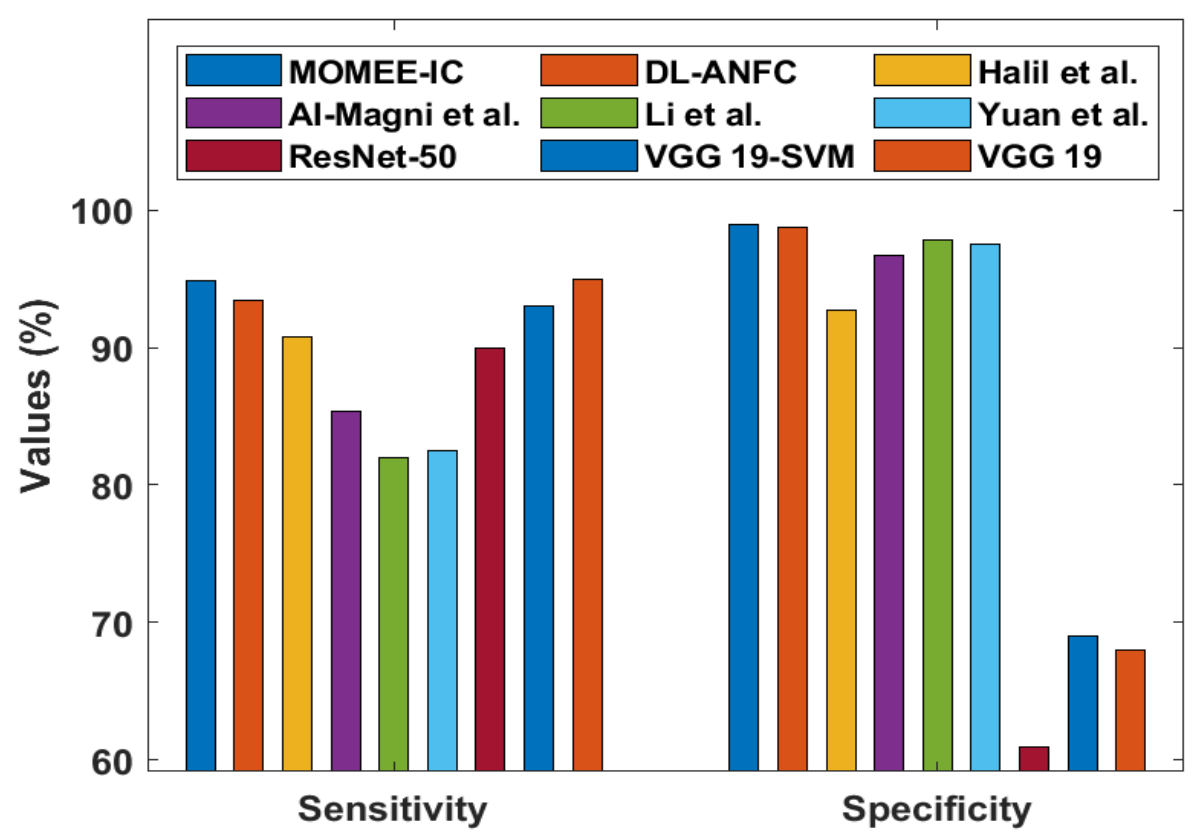

Fig. 10. Comparative analysis of MOMEE-IC Method interms of sensitivity and specificity.

\section{Conclusion}

This paper has devised a novel model for secure medical image transmission and classification in Big Data Environment. The presented model involves two stages such as encryption and image classification. Initially, the medical images are encrypted using the MHE-LOA algorithm where the optimal keys in the MHE method are generated using the LOA. Next, the encrypted images will be transmitted to the cloud using the big data environment. Upon reception, the decryption of medical images takes place, and then image classification process gets executed using SDAE-LR model. For ensuring the optimal results of the presented method, an extensive results analysis takes place and the outcomes are examined in distinct aspects. The obtained simulation result verified the betterment of the presented algorithm interms of security and detection rate.

\section{References}

[1] Mehta, N.; Pandit, A. Concurrence of big data analytics and healthcare: A systematic review. Int. J. Med. Inform. 2018, 114, 57-65.

[2] Wu, X.; Zhu, X.; Wu, G.-Q.; Ding, W. Data mining with big data. IEEE Trans. Knowl. Data Eng. 2014, 26, 97-107.

[3] Dinov, I.D. Volume and Value of Big Healthcare Data. J. Med. Stat. Inf. 2016, 4. pii: 3.

[4] Morris, M.A.; Saboury, B.; Burkett, B.; Gao, J.; Siegel, E.L. Reinventing Radiology: Big Data and the Future of Medical Imaging. J. Thorac. Imaging 2018, 33, 4-16.

[5] Ristevski, B.; Chen, M. Big Data Analytics in Medicine and Healthcare. J. Integr. Bioinform. 2018, 15.

[6] Choy R, Edelman A. Parallel MATLAB: doing it right. Proc IEEE. 2005;93(2):331-341.

[7] Li W, Feng C, Jin C, Chen Q, Liu H, Zhao D. A two-level cloud storage system based on asynchronous message for medical image big data. Paper presented at: Proceedings of the 2019 5th International Conference on Big Data Computing and Communications (BIGCOM), QingDao, China, China; 2019.

[8] Meena K, Sujatha J. Reduced time compression in big data using mapreduce approach and hadoop. J Med Syst. 2019;43(8):239.

[9] Alias N, Suhari NNY, Saipol HFS, et al. Parallel computing of numerical schemes and big data analytic for solving real life applications. J Teknol. 2016;78(8-2).151-162.

[10] Chrisochoides N, Fedorov A, Kot A, et al. Toward real-time image guided neurosurgery using distributed and grid computing. Paper presented at: Proceedings of the 2006 ACM/IEEE Conference on Supercomputing, Tampa Florida; 2006:76-es.

[11] Augustine D, Peter RP. Performance evaluation of parallel genetic algorithm for brain MRI segmentation in hadoop and spark. Ind J Sci Technol. 2016;9(48):1-7. https://doi.org/10.17485/ijst/2016/v9i48/91373.

[12] Patil JS, Predeepini Dr G. Two dimensional medical images diagnosis using MapReduce. Ind J Sci Technol. 2016;9(17).1-5.

[13] Patil JS, Pradeepini G. Three-dimensional MRI brain image analysis on hadoop platform. Paper presented at: Proceedings of the International Conference on Intelligent Computing and Applications; 2018:131-142; Springer, Singapore.

[14] Shankar, K., Lakshmanaprabu, S.K., Gupta, D., Khanna, A. and de Albuquerque, V.H.C., 2020. Adaptive optimal multi key based encryption for digital image security. Concurrency and Computation: Practice and Experience, 32(4), p.e5122.

[15] Yazdani, M. and Jolai, F., 2016. Lion optimization algorithm (LOA): a nature-inspired metaheuristic algorithm. Journal of computational design and engineering, 3(1), pp.24-36.

[16] Bezdek JC (2013) Pattern recognition with fuzzy objective function algorithms. Springer, Berlin

[17] Xing, C., Ma, L. and Yang, X., 2016. Stacked denoise autoencoder based feature extraction and classification for hyperspectral images. Journal of Sensors, 2016.

[18] Shankar, K., Lakshmanaprabu, S.K., Gupta, D., Khanna, A. and de Albuquerque, V.H.C., 2020. Adaptive optimal multi key based encryption for digital image security. Concurrency and Computation: Practice and Experience, 32(4), p.e5122.

[19] Sikkandar, M.Y., Alrasheadi, B.A., Prakash, N.B., Hemalakshmi, G.R., Mohanarathinam, A. and Shankar, K., 2020. Deep learning based an automated skin lesion segmentation and intelligent classification model. Journal of ambient intelligence and humanized computing, pp.1-11. 